\title{
Dati storici sulla presenza circummediterranea del francolino nero Francolinus francolinus francolinus (Linnaeus, 1766)
}

\author{
Aldo Oriani
}

\begin{abstract}
Riassunto - Si espone la distribuzione storica del francolino nero sulle coste del Mediterraneo; da quelle asiatiche, dove era endemico, venne introdotto e si naturalizzò in Grecia fin dall'antichità e dal XIII secolo in Sicilia ed Aragona. In quel secolo ne parlò Federico II di Svevia nel suo trattato di falconeria dimostrandone una approfondita conoscenza e contemporaneamente la specie veniva citata nelle leggi dell'Aragona. Da queste due regioni il francolino venne diffuso in ampie zone della Spagna, della Francia sud-occidentale, della Toscana e forse anche della Lombardia ed è emersa l'ipotesi che la specie fosse presente anche in Egitto.

Il francolino scomparve, tra la fine del Settecento e la metà dell'Ottocento, da tutti i territori dove era stato introdotto a scopo venatorio a causa delle modifiche ambientali conseguenti alle bonifiche e alla antropizzazione e della caccia non regolamentata, ormai non più prerogativa esclusiva della nobiltà che, per secoli, si era adoperata a tutelare la specie. Analoghe cause portarono, nel corso dell'Ottocento, alla scomparsa del francolino anche da quasi tutti gli ambienti costieri del Mediterraneo orientale, tanto che, già negli anni Trenta del secolo scorso, si nutrivano preoccupazioni sulla sua salvaguardia anche nei territori dove era indigeno.
\end{abstract}

Parole chiave: Francolino nero, Mediterraneo, distribuzione storica.

Abstract - Historical data on the circummediterranean distribution of the Black Francolin Francolinus francolinus francolinus (Linnaeus, 1766).

This study presents the historical distribution of Black Francolin along the coasts of the Mediterranean Sea; from eastern coastlines, where it was endemic, the Black Francolin was introduced and naturalized from ancient times in Greece, and, starting from the $13^{\text {th }}$ Century, in Sicily and Aragon. In that period, Federico II of Svevia in his essay on falconry demonstrated a deep knowledge of the topic and, at the same time, the species was cited in the Aragon Laws. From these areas, the Black Francolin was brought to Spain, South-Western France, Tuscany, and possibly in Lombardy. Later on, it was suggested that the species was present also in Egypt.

Due to environmental changes - caused by the increasing presence of humans and the measures taken to reclaim territories plus the unregulated hunting - the Black Francolin disappeared, between the end of

Società Italiana di Scienze Naturali - Centro Studi Storiconaturalistici, corso Venezia 55, 20121 Milano (MI) Italia E-mail: aldor17@libero.it

(c) 2014 Aldo Oriani

Received: $11^{\text {th }}$ November 2014

Accepted for publication: $27^{\text {th }}$ November 2014 the $18^{\text {th }}$ and the first half of the $19^{\text {th }}$ Century, from those areas where it had been introduced for hunting. This activity, in fact, was no longer considered as a privilege of high society, which for years took care of the bird. During the $19^{\text {th }}$ Century, similar reasons led to the disappearance of the Black Francolin also from many Eastern Mediterranean shorelines. This is why, from the Thirties of the last Century, there were worries about its safety, even in those areas where it was native.

Key words: Black Francolin, Mediterranean, historical distribution

\section{IL NOME}

Nel latino classico il francolino veniva definito atta-

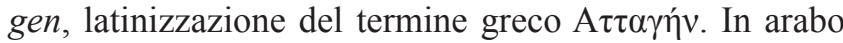
la specie è detta hajal aswad (Andrews, 1995: 75), vale a dire pernice nera, radice che ha influenzato unicamente il maltese. In russo, turco, azero ed armeno la radice è " $t u$ $r a$ ", mentre, comune a quasi tutte le lingue europee ${ }^{1}$, è la radice "francolin", utilizzata fin dal secolo XIII in Sicilia ed in Aragona. L'origine di quest'ultima appare recente e non derivata né dall'arabo né dalle lingue classiche.

Il nome scientifico "francolinus" è frutto della latinizzazione del nome italiano (Moltoni, 1946): Federico II di Svevia, nel XIII sec., nel «De arte venandi cum avibus», utilizza il termine "franquilinus" e, più raramente, "francuillinus" perché: "quando Noi non abbiamo saputo trovare nella lingua latina $i$ termini appropriati ad ogni situazione, ci siamo serviti di quelli che Ci sono sembrati esser più vicini a fare comprendere il nostro pensiero" (Trombetti Budriesi, 2000: 5) implicitamente confermandoci che il termine era già in uso nel volgare.

L'Olina (1622: 33) riconduceva il termine alla: "franchezza del viver, che ha rispetto alle bandite, e rigorosi editti che per conto di quello da Principi si fanno. Il nome di Franguellina è certissimo, che è corrotto dal suddetto, essendo facilissimo da Francolino, ò Frangolino cader in dir Franguellino" ed il Tanara (s.d.), nello stesso secolo, ne spiegava così l'etimologia: "i principi con bandi proibiscono che siano ammazzati, il qual asilo, o vogliam dire franchigia, godendo questi uccelli, fu causa che fossero chiamati francolini". Sembra però più corretto ritenere che il termine derivi dai crociati "franchi" che lo importarono dalla Terra Santa e lo diffusero nei regni cristiani del Mediterraneo centroccidentale (Jiménez, 2013: 22).

Molti autori, tra il Quattro e il Settecento, citarono il francolino, ma non sempre è chiaro a quale specie si ri- 
ferissero. Spesso il termine è impropriamente usato per il francolino di monte (Bonasa bonasia), le pernici del genere Alectoris, le grandule del genere Pterocles, e la gallina prataiola (Tetrax tetrax). Olina (1622: 33), tra varie osservazioni corrette, ne riporta altre riferibili alla pernice sarda (Alectoris barbara): "Trovasi copiosamente in Barbaria massime presso a Tunis, dond'è che qualch'uno gl'ha dato nome di Pernice di Barbaria" ed al francolino di monte: "Què pochi ch'apparisco in Italia vengon dall'Alpi". Le specie talvolta non sono ben identificabili, in quanto il nome "è parimente uno di quelli che sono stati applicati ad uccelli molto diversi. [...] era stato dato all'attagas, e da un passo di Gessner sembra che l'uccello noto in Venezia sotto il nome di francolino, sia una specie di gallina selvatica (hazel-huhn). Il francolino di Napoli è più grosso d'una gallina ordinaria, e a dire vero, la lunghezza de suoi piedi, del suo becco e del suo collo, non permettono di farne né una gallina selvatica, né un francolino. Tutto ciò che dicesi del francolino di Ferrara, si è che ha i piedi rossi e vive di pesci. L'uccello di Spitzberg, a cui si è dato il nome di francolino, si chiama anche corridore di spiaggia poiché non s'allontana mai di molto dalla costa ove trova il convenevol nodrimento [...] ma non è più grosso d'un'allodola" (Buffon, 1775: 175-176). Ancora nei primi decenni dell'Ottocento si perpetuava la confusione: veniva tradotto con "attagaso" il termine inglese "red grous" (Smith, 1826: 28) ed il "francolin" si diceva fosse di passo accidentale primaverile nel nizzardo (Sceembri, 1843: 19). Ancora oggi popolarmente il termine identifica nei Paesi Baschi l'occhione (Burhinus oedicnemus) e in altre zone spagnole dei limicoli migratori (Jiménez, 2013: 17). Lavauden (1936: 303-305), nella accurata ricognizione su quanto scritto in passato sulla specie, concluse che: "queste ricerche sono rese estremamente difficili per l'inestricabile confusione che ha regnato tra gli autori antichi, dove il nome di Francolino è stato utilizzato per designare uccelli del tutto diversi. L'identità degli uccelli designati varia anche non soltanto con gli autori, ma perfino a livello di singolo naturalista".

\section{LA STORIA}

Agli antichi greci la specie era ben nota, non disdegnavano affatto la pratica della caccia agli uccelli e soprattutto apprezzavano le carni del francolino (Garcia Soler, 2003) che viveva nelle pianure costiere da Megaris alla Beozia e su alcune isole dell'Egeo.

I Romani identificarono il francolino col termine greco latinizzato "attagen ionius" e con questo termine è citato, per la squisitezza delle sue carni, da Orazio, nel I secolo a.C., e da Marziale, nel I d.C. (Vivaldi, 1993: 688). Plinio afferma che "si trova in Gallia, in Spagna ed anche sulle Alpi" (Cuvier, 1828: 313), evidenziando però la confusione col francolino di monte. Il francolino nero è dipinto in una villa ad Oplontis, sepolta dall'eruzione del Vesuvio nel 79 d.C., ed è raffigurato nei mosaici di una villa ad El Djem in Tunisia, datati al II-III secolo d.C. (Arnott, 2007: 32). Tutte le testimonianze romane sono successive al II secolo a.C. e quindi alla conquista della Grecia, suggerendo l'ipotesi che il francolino fosse parte del più ampio processo di grecizzazione dei costumi latini che seguì l'annessione: ad ulteriore conferma, il termine latino è la letterale trascrizione di quello greco. In Sicilia probabilmente non era presente, infatti, nonostante l'isola fosse stata conquistata circa un secolo prima della Grecia, la specie non è menzionata nei testi latini coevi. In ambito romano il francolino veniva esclusivamente allevato a scopo alimentare e, dato che la caccia non era considerata un'attività nobile, ma era concepita unicamente come impegno servile (Galloni, 1993), mancavano i presupposti perché si procedesse a una sua introduzione a fini venatori.

Nel medioevo il francolino, assieme ai fagiani e alle pernici, era una delle prede d'eccellenza dell'astoreria, la caccia di "volo basso" con l'uso degli Accipitridi. La falconeria in senso lato, compresa quella di "volo alto" con l'utilizzo di Falconidi, era una prerogativa della nobiltà e forse questo fu il presupposto dell'introduzione della specie nei regni cristiani del Mediterraneo centrooccidentale.

Federico II di Svevia (1194-1250) conosceva bene il francolino e lo citò più volte nel suo trattato sulla falconeria, non con un dotto termine classico né con uno di origine araba, ma come franquilinus o francuillinus. Il fatto che la specie avesse un nome in volgare latinizzato forse si può spiegare con una sua ampia diffusione anche al di fuori delle riserve nobiliari. Non sussistono dubbi sulla esatta identificazione nei diversi capitoli ed è anche raffigurata correttamente nelle miniature dei manoscritti (Trombetti Budriesi, 2000). In quegli stessi anni le leggi del Regno d'Aragona del 1247 includevano il francolino tra le specie cacciabili (Jiménez, 2013: 17). Vi furono quindi almeno due diversi nuclei di introduzione nel Mediterraneo centro-occidentale anteriori alla metà del XIII secolo: uno siciliano, forse più antico, e l'altro aragonese. Dal XIV secolo si registra un notevole ampliamento dell'areale in correlazione con l'espansione CatalanoAragonese nel Mediterraneo: la specie, già presente in Aragona e nei suoi possedimenti siciliani e greci, i ducati di Atene e Neopatria, venne ampiamente diffusa a fini venatori nei territori sotto il suo controllo.

\section{LA DISTRIBUZIONE}

Come si è visto, gli scritti naturalistici del passato vanno vagliati con estrema cura prima di poter affermare che il francolino fosse realmente presente in un territorio. I dati che seguono sono emersi da un'attenta analisi dei testi che evidenziano una precisa conoscenza della specie dal punto di vista comportamentale ed ambientale e che riferiscono con cognizione sia del francolino che delle specie generalmente con esso confuse. La più elevata attendibilità delle fonti si riscontra negli scritti di carattere venatorio: sia i cacciatori sia le autorità preposte alla protezione della selvaggina denotano una perfetta conoscenza della specie.

Il francolino nero, Francolinus francolinus (Linnaeus, 1766) ha, ancor oggi, una distribuzione molto vasta: dalle sponde orientali del Mediterraneo al confine Indo-Birmano (Forcina et al., 2012: 524). In questo vastissimo areale sono state identificate sei sottospecie (McGowan, 1994: 489), di cui quella nominale, oggetto di questa ricerca, è 
tuttora presente a Cipro, in Anatolia, Israele, Giordania, Siria, Iraq, Transcaucasia ed Iran settentrionale.

Nel Pleistocene, in Europa, erano presenti altre specie del genere Francolinus, ma $F$. francolinus è stato rinvenuto solo in Israele, Siria e Iraq (Tyrberg, 1998). Il francolino nero è autoctono sulle coste mediterranee dell'Asia e, fino alla prima metà dell'Ottocento, le popolazioni costiere dal Mar di Marmara alla Palestina, dove le condizioni ambientali lo consentivano, non erano disgiunte da quelle delle zone interne mediorientali. La specie era probabilmente autoctona anche sulle isole di Lesbo, Samo, Rodi e Cipro.

La Sardegna non sembra abbia mai ospitato il francolino (Lilford, 1862: 353; Arrigoni Degli Oddi, 1929: 808; Lavauden, 1936: 307; Mayaud, 1939: 67; Maluquer \& Travé, 1961: 134) anche se taluni lo hanno affermato (Temminck, 1815: 347; Dresser, 1877: 3; Ogilvie-Grant, 1896: 104; Brichetti, 1985: 24).

In Corsica, nonostante alcuni autori (Lavauden, 1936: 307; Perco, 1981) ne citino la presenza fino ai primi decenni del XIX sec., non è emersa alcuna prova della sua esistenza (Mayaud, 1939; Maluquer \& Travé, 1961: 134).

A Malta la specie è citata da Latham (1783: 760), ma l'isola sembra sia "la località più improbabile" per la specie (Lilford, 1862: 353) ed altri hanno confermato che non è emerso alcun riscontro della sua presenza storica (Sceembri, 1843: 19; Sultana et al., 1975). Nel corso di questa ricerca è stato tuttavia rilevato che, nella prima metà del Settecento, ve ne giunsero dall'Egitto e vennero poi inviati in dono allo scienziato francese Ferchault de Réaumur (Brisson, 1760: 247).

In Africa nordoccidentale il Francolinus francolinus non sembra sia mai vissuto; le numerose affermazioni di presenza (Buffon, 1775: 178; Latham, 1783: 760; AA.VV., 1784: 50; Mouton-Fontenille, 1811: 352; Temminck, 1815: 347; Ogilvie-Grant, 1896: 104; Arrigoni Degli Oddi, 1902: 338; Perco, 1981) sono molto dubbie (Arrigoni Degli Oddi, 1929: 806) e probabilmente del tutto infondate (Lavauden, 1936: 305-306). L'equivoco potrebbe essere stato originato dalla presenza in zona della pernice sarda, degli pteroclidi e del francolino bisperonato dell'Atlante marocchino (Francolinus bicalcaratus ayesha Hartert, 1917) o anche di taluni limicoli che venivano identificati con questo nome (Shaw, 1743: 330).

Per quanto concerne l'Egitto, il francolino pare fosse ignoto agli Egizi (Houlihan, 2002) e, nella seconda metà del Settecento, vi furono contrastanti opinioni riguardo alla sua presenza. Alcuni attenti viaggiatori (Sonnini, 1798) non lo menzionarono tra gli uccelli del Delta del Nilo, che pure era un ambiente favorevole alla specie; altri invece ne affermarono la presenza (Buffon, 1775: 178; Latham, 1783: 760; Moutton-Fontenille, 1811: 352). A gettare una nuova luce sulla questione sono emersi interessanti riscontri: in almeno due occasioni dall'Egitto giunsero in Europa dei francolini: "Il signor Hollande, dottore in medicina, ne ha riportati molti da quest'ultimo paese; sono un po' più grandi di quello che ho descritto [francolino nero] e non mi sono sembrati differenti per altro" (AA. VV, 1774: 50), altri ne furono inviati a Malta "al signor Godeheu, che in seguito li ha donati al signor de Reau- mur" (Brisson, 1760: 247). Queste informazioni sembra siano sfuggite ai naturalisti ed i più ritennero che il francolino non fosse mai appartenuto alla fauna egiziana (Lavauden, 1936: 306).

\section{IL MEDITERRANEO ORIENTALE}

Originariamente il francolino nero era autoctono sulle coste mediterranee dell'Asia e la sua distribuzione non era disgiunta dalle zone più interne. Nel corso degli ultimi due secoli l'areale si è fortemente contratto e frazionato in distretti di rifugio del tutto isolati geograficamente gli uni dagli altri e le popolazioni residue sono andate riducendosi tanto che già nei primi decenni del secolo scorso erano emerse preoccupazioni sulla salvaguardia della specie (Lavauden, 1936: 309). Gli areali più prossimi a quelli costieri, ma ormai disgiunti, sono lungo il medio corso dell'Eufrate in Siria (Hollom et al., 1988: 76) e sul confine turco-siriano tra il Tigri e l'Eufrate (Kirwan et al., 2008: 52)

In Turchia era presente sul Bosforo fino alla metà dell'Ottocento (Kirwan et al., 2008: 52) e lungo le coste dell'Egeo, era abbondante nelle paludi e lungo il fiume Gediz (Labat, 1735: 64) ed anche lungo il corso del Menderes e delle sue convalli dalla foce fino a Pamukkale. Già dalla seconda metà del secolo però andava scomparendo da quasi tutti questi luoghi esclusi la foce del Menderes e la valle di Arpaz, sua tributaria (Dresser, 1877: 2). Nei dintorni di Izmir nidificò almeno fino al 1899 (Kirwan et al., 2008: 52). Oggi è del tutto scomparso dalle coste dell'Egeo, dalle valli del Dalaman, del Menderes e dalle rive del Lago Köyceğiz, nell'entroterra di Marmaris, dove visse fino al 1960 (McGowan, 1994: 489; Kirwan et al., 2008: 52). Ormai sopravvive solo in due distinte aree della costa della Cilicia: nei delta dei fiumi Ceyhan e Seyhan, tra Mersin e Yumurtalik, e lungo il fiume Goksu; al di fuori di queste due zone alcune aree protette garantiscono la sopravvivenza di altri piccoli nuclei (Martins, 1989; Berk, 1991; Kirwan \& Martins, 1994; Kirwan et al., 2008: 52). Sulle sponde settentrionali del lago di Antiochia viveva una popolazione, geograficamente isolata, ora estinta, caratterizzata dal colorito scuro, che venne descritta come ssp. billypayni (Meinertzhagen, 1933: 21).

Agli inizi dell'Ottocento il francolino era presente anche lungo tutta la costa mediterranea da Antiochia a Tel Aviv. Nel corso di quel secolo scomparve dalla Siria settentrionale e dalla regione di Aleppo (Lavauden, 1936: 309), ma era ancora numeroso in Libano nei dintorni di Tripoli (Volney, 1801: 225) e di Beirut (Lilford, 1862: 356). Più a sud lungo la costa era ampiamente diffuso, anche se non abbondante, ad Acri, Haifa, Cesarea, nella piana di Sharon e nella zona di Tel Aviv (Volney, 1801: 225; Wilde, 1840: 165; Tristram, 1865: 91; Tristram, 1884: 124). Le popolazioni costiere scomparvero con la progressiva messa a coltura del territorio, ma sopravvissero quelle delle contigue zone interne nella piana di Esdraelon e lungo la valle del Giordano. A fine Ottocento e nei primi decenni del Novecento il francolino era numeroso lungo il Giordano dalla valle di Hula, lungo le sponde del lago di Tiberiade, fino a Gerico (Tristram, 1865: 435; Tristram, 1868; Tristram, 1884: 124; Festa, 1894: 9; Mei- 
nertzhagen, 1920). La specie si estinse in Libano nel 1958 (Ramadan-Jaradi \& Ramadan-Jaradi, 1999). Attualmente è ancora presente in Galilea, nel nord della Samaria (McGowan, 1994: 489; Forcina et al., 2012: table S1) ed in Giordania nella valle del Giordano e sulla costa sudorientale del Mar Morto (Andrews, 1995: 75).

Nell'antica Grecia, dove probabilmente era stata importata dalla Lydia, la specie era presente in Attica, nella piana di Maratona, in Beozia e nelle piane di Megaris (St. John, 1844: 152; Arnott, 2007: 32, 282). Intorno al 1850 era rara nelle zone con alte erbe in prossimità delle coste della Macedonia (Drummond, 1846: 13; Lilford, 1862; 354). Nel Settecento viveva sulle isole di Samo (Pitton de Tournefort, 1718: 159; Brisson, 1760: 247; MoutonFontenille, 1811: 352) e di Lesbo, ma nei primi decenni dell'Ottocento vi era ormai scomparsa (Lilford, 1862: 354). A Rodi (Mouton-Fontenille, 1811: 352) il francolino era ancora comune intorno al 1850 , ma poi rapidamente scomparve. È infine possibile che sia stato presente anche a Creta (Tristram, 1884: 124; Masseti, 2008: 212).

A Cipro (Brisson, 1760: 247; Mouton-Fontenille, 1811: 352) nel Settecento era uno degli uccelli più comuni: veniva citato col nome italiano o come Aftokinara (Pococke, 1745: 231) e lo si vendeva nei mercati ad un prezzo simile a quello delle pernici (Mariti, 1769: 31). Ancora nella seconda metà dell'Ottocento viveva nella penisola di Karpas, sulle colline ad ovest di Trikomo (Lilford, 1875) e nella zona di Pafos (Lilford, 1862: 355), ma cominciava a diventare sempre più raro (Malherbe, 1843: 151). All'inizio del secolo scorso la situazione peggiorò drasticamente e si rese necessaria, per garantirne la sopravvivenza, la completa interdizione della caccia per un decennio, tuttavia, intorno al 1930, il francolino scomparve dalla piana di Morfou, dal litorale di Limassol e dall'alta valle di Chrysochou e nei decenni successivi cominciò a scarseggiare anche a capo Kornatikis, a nord di Morfou
(Bannerman \& Bannerman, 1958). L'areale si ridusse a due zone distinte: una occidentale, il litorale di Pafos, la penisola di Akamas e la piana di Polis; l'altra orientale, la penisola di Karpas (Hüe \& Etchécopar, 1970: 222). A Cipro queste due zone ancora oggi sono le roccaforti della specie (Boye, 1990; Roberts, 1999) che vive nei bassipiani costieri spingendosi nelle vallate anche a qualche decina di chilometri dalla costa. L'habitat preferito sono le zone cespugliose ed i margini dei boschi al limitare delle praterie, normalmente presso corsi d'acqua con alte erbe nelle loro adiacenze, pur non disdegnando anche $\mathrm{i}$ campi di cereali, di ortaggi e le vigne se offrono adeguate zone di riparo e di fuga. Recentemente la popolazione è in espansione grazie alla liberazione di individui allevati in cattività: le uova vengono prelevate nelle riserve dove la densità è più elevata $\mathrm{o}$ nelle zone agricole dove $\mathrm{i}$ nidi andrebbero distrutti durante il raccolto, i rilasci vengono effettuati in riserve di caccia con ambienti idonei lungo la costa dove un tempo la specie era presente (Fig. 1) (www. kypros.org/Cyprus/francolin.htm).

\section{LA SICILIA}

Tutti gli autori concordano nel ritenere che il francolino non fosse indigeno in Sicilia (Martorelli, 1906: 20; Baldacci, 1964) anche se alcuni non escludono che potesse essere autoctono e "siccome non si ha il minimo documento circa il tempo da cui datava la presenza del Francolino in Sicilia e potrebbe quindi trattarsi di un tempo remotissimo, sorge anche l'ipotesi che sia uno dei tanti effetti di isolamento dovuti a cause geologiche" (Martorelli, 1906: 20). Come già evidenziato, si è escluso che il francolino vivesse in Sicilia all'epoca della conquista romana e, alla luce di quanto si conosce, la sua presenza non è neppure correlabile alla dominazione araba protrattasi dall' 827 al 1072.

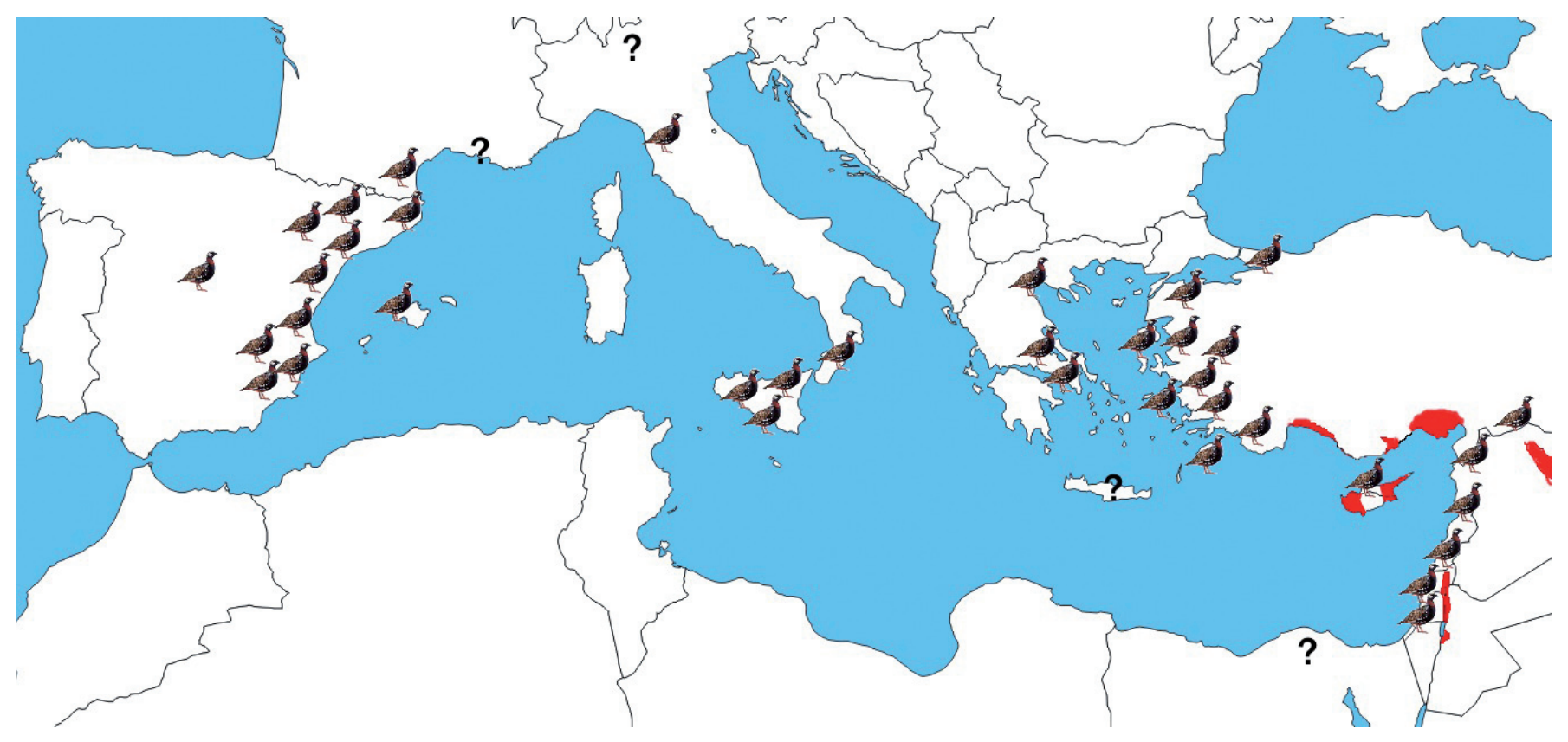

Fig. 1 - Distribuzione circummediterranea del francolino nero: attuale ( $\square$ ), storica (\$), non sufficientemente documentata (?) / Circummediterranean distribution of the Black Francolin: present ( $\square$ ), historical (\$), insufficient information (?). 
La più antica testimonianza è quella di Federico II di Svevia (1194-1250), che non ci risulta sia mai stata presa in considerazione nei precedenti lavori dedicati al francolino. Federico da giovane apprese, alla corte di Palermo, la falconeria e l'astoreria, e scrisse, del francolino, con estrema competenza nel primo libro "De arte venandi cum avibus" la cui redazione, probabilmente del figlio Manfredi re di Sicilia, è databile al 1258-1266 (Trombetti Budriesi, 2000). Pur utilizzando il latino, non viene mai usato il termine classico "attagen", ma esclusivamente quello, di probabile origine volgare, "franquilinus" o "francuillinus". Federico annotò che si cibava, come gli altri galliformi, di "grana, fructus arborum et dumorum, brucos et cetera convenientia sibi" (cap. 32); che essendo un modesto volatore si recava all'alba in pastura per poi rintanarsi nei cespugli e uscire di nuovo solo verso il tramonto (cap. 38); che era preda dello sparviere e del lodolaio come altri Galliformi e Columbiformi (cap. 58); che volava battendo le ali con frequenza (cap. 256 e 299); che il suo volo, essendo diritto e breve, lo rendeva facile preda dei rapaci che lo inseguivano, e che la sua scarsa resistenza al volo consentiva di catturarlo anche con le mani o con i cani (cap. 263) (Trombetti Budriesi, 2000). La testimonianza di Federico è in linea con quanto emerso dalle ricerche archeozoologiche che hanno datato al XIII secolo i più antichi reperti ossei di francolino rinvenuti in Sicilia nel sito di Calathamet, non lontano da Alcamo (Masseti, 2009: 18).

Si può quindi ritenere che l'introduzione della specie sull'isola sia conseguente alle crociate, peraltro Federico stesso era stato in Terra Santa tra il 1228 ed il 1229 durante la sesta crociata. Anche la tradizione isolana vuole che siano stati i crociati ad introdurla giungendo perfino a identificare gli ordini dei templari e dei teatini (Amari, 1937), ordine questo costituito nel 1524 e che quindi non può aver avuto parte in quegli eventi. Non condivisibile invece l'ipotesi che il francolino sia stato introdotto in Sicilia dagli aragonesi, che si impossessarono dell'isola solo nel 1282, ma non si può tuttavia escludere che vi siano state ulteriori immissioni nei secoli successivi, durante il loro dominio.

Nel Cinquecento sono molti i riscontri di presenza: norme emesse dalle autorità spagnole, particolarmente attente alla tutela della specie che facilmente poteva essere eradicata da una caccia non regolamentata. La prammatica del 10 marzo 1550 del viceré Juan de Vega prese atto che molti predavano i francolini con lacci, reti ed altri sistemi e che così in breve tempo la specie sarebbe scomparsa e bandì quindi la caccia, esclusa quella con i falchi, minacciando per i trasgressori la "pena onze 200 in pro dell'erario in ogni trasgressore, o di galea ad arbitrio del Governo qualora il reo fosse persona di basso rango" ammenda oltremodo elevata peraltro in linea con la severa pena alternativa. Nel 1565 il viceré García Àlvarez de Toledo riconfermò le precedenti disposizioni che vennero nuovamente ribadite nel 1568 dal viceré Francesco Ferdinando Avalos (Amari, 1937). Alla fine del secolo il Crivella (1593) inviò una relazione alla corte di Madrid nella quale citava, tra le specie di interesse venatorio della Sicilia, il francolino assieme a pernici, caprioli e cervi.
Nel Seicento sull'isola ve ne erano "molti" (Olina 1622: 33) e forse era anche "abbondante" (Falcone, 1675: 221). Lo si rinveniva dove l'ambiente era idoneo: lungo la costa tirrenica messinese ad Acquedolci, nell'entroterra palermitano, a Misilmeri (PA), e nella vasta area compresa tra Scordia (CT), Caltagirone (CT), Piazza Armerina (EN) ed il litorale tra Palma di Montichiaro (AG) e Gela (CL) (Amari, 1937: 1083). In quel secolo Tanara (s.d.) lo cacciò e verificò la facilità di colpirli durante i suoi brevi voli in linea retta aggiungendo che era chiamato anche "Predice Asculapica" per le supposte proprietà terapeutiche delle sue saporite carni ritenute "molto a proposito per quelli che hanno lo stomaco debole, e quelli che patiscono dolore di renella". Già nel secolo precedente il viceré de Vega aveva definito i francolini "utili in molte occorrenze per la salute dei corpi umani” (Amari, 1937), anche Olina (1622: 33) ne aveva elogiato "oltre l'esquisitezza del sapore buoni per la sanità giudicandosi le sue carni molt'a proposito per quelli che hanno lo stomaco debole, ò patiscono di Renella, ò pietra" ed ancora nel secolo successivo ne venivano elencate le presunte proprietà terapeutiche (James, 1753: 500-501). Nel Sei-Settecento i proprietari dei latifondi, spesso incolti, ne favorirono la diffusione ed anche nelle sue ultime roccaforti nei dintorni di Gela, vi fu immesso a scopo venatorio (Carvana 1889).

I Borboni, che governarono l'isola dal 1734, confermarono la protezione ed istituirono premi per la tutela dei nidi e delle covate. Nel 1783 il viceré Domenico Caracciolo proibì la caccia alla specie nei periodi di residenza del re in Sicilia (Amari, 1937: 1086). In quegli anni Latham (1783: 760) affermava che era presente a Lipari, ma l'informazione non sembra corretta: Spallanzani, nel 1793, menzionava, su quell'isola, la "pernice", in realtà la coturnice (Alectoris graeca). Questa scomparve da Lipari alla fine dell'Ottocento e, dopo qualche decennio, anche da Salina prima che fosse possibile accertare se si trattasse di una sottospecie endemica o soltanto di una popolazione isolana della sottospecie whitakeri Schiebel, 1934 (Moltoni \& Frugis, 1967: 120).

Intorno al 1780 il francolino non era raro attorno a Palermo (Sonnini, 1798: 54) e re Ferdinando, nel 1800, contribuì a diffonderlo ulteriormente introducendolo con successo nel parco della reale villa la Favorita alle pendici del Monte Pellegrino (Doderlein, 1869: 159), erano anni in cui se ne uccidevano anche nei dintorni della città (Lilford, 1862: 356). Lungo la costa settentrionale dell'isola viveva nei piani del Finale, a ovest di Cefalù (PA), ed in varie località delle Madonie da dove scomparve nei primi anni del secolo (Minà-Palumbo, 1889: 521). In quei decenni, nonostante l'areale cominciasse a contrarsi, era ancora abbastanza comune nella zona tra Castelvetrano (TP), Partanna (TP) e Sciacca (AG) ed in quella compresa tra Licata (AG), Gela (CL) e Caltagirone (CT), e popolamenti più ridotti sopravvivevano a Misilmeri (PA) e Mortelle (ME) (Doderlein, 1869: 159; Salvadori, s.d.: 187). Lungo la costa ad ovest di Palermo la specie scomparve intorno al 1860 (Orlando, 1936: 204), nelle regie bandite siciliane era divenuta scarsa già nella prima metà del secolo (Costa, 1857: 55-56). Nei primi decenni dell'Ottocento nelle pianure tra Caltagirone e Gela (Malherbe, 
1843: 151; Sceembri, 1843: 19) diventava sempre più raro "per l'abuso della caccia che si esercita in tutti i tempi e in tutte le stagioni" (Benoit, 1840: 118), lo si incontrava però anche nelle vicinanze dell'abitato di Gela dove arrivava pedonando lungo le rive, coperte da canneti e cespugli, dei fiumi Gela e Cimia (Carvana, 1889: 516). Era presente anche nelle contigue aree collinari di Butera e di Mazzarino ${ }^{2}$ e lungo la costa verso Licata dove, poco prima del 1850, se ne uccidevano molti. L'ambiente naturale di questi ultimi distretti di rifugio avrebbe consentito la sopravvivenza della specie: tra Piazza Armerina (EN) e Gela vi erano poche coltivazioni, l'habitat era perfetto per il francolino e la vegetazione lungo i corsi d'acqua ne favoriva la diffusione (Amari, 1937). A ovest di Falconara e a est di Gela, dove pure vi erano ambienti idonei, mancavano però corridoi naturali di vegetazione rendendo impossibile la propagazione della specie (Carvana, 1889: 519-520). Le bonifiche, la caccia insensata al tempo delle covate e il prelevamento delle uova in pochi anni fecero tracollare le popolazioni nonostante la protezione accordata delle leggi (Carvana, 1889: 516-518; Amari, 1937).

Intorno al 1850 il francolino era diventato raro e sopravviveva in due distinti distretti di rifugio: il più vasto tra Licata, Gela, Butera e Caltagirone, l'altro nel trapanese. Lilford (1862: 352), condusse ricerche in queste località nel 1856 e nel 1858, ma non ne rinvenne nonostante a Gela se ne abbattessero nel 1858 (Minà-Palumbo, 1889: 521 ) ed intorno al 1860 ve ne fossero ancora (Doderlein, 1869: 159). Sette esemplari, uccisi tra il 1850 ed il 1862, confluirono nelle collezioni del Museo di Firenze ed una femmina, uccisa nel 1863, in quelle di Torino (Arrigoni degli Oddi, 1929: 807). Nel 1864 venne abbattuta una coppia a Desusino, tra Gela e Licata (Carvana, 1889: 518 ) ed ancora nel 1865 ne furono uccisi a Suero e Butera (Doderlein, 1869: 160). L'ultimo francolino, di cui si ha notizia, venne abbattuto nell'autunno 1869 nella tenuta di Falconara, presso Gela, e finì "imbandito in un pranzo" (Doderlein, 1869: 160).

Era ormai praticamente estinto quando si aprì un vivace scambio di opinioni tra gli ornitologi sulla sua sopravvivenza. Il Saunders (1869: 396) aveva appreso che era ancora presente a Gela, ma aveva dubbi: era stato in Sicilia ed aveva offerto una cospicua somma per averne uno in carne, ma senza successo. Salvadori (1869) controbatté asserendo che Dordelein ne aveva ricevuti di recente al Museo dell'Università di Palermo. Saunders (1870) rispose sarcastico che, fino al febbraio 1869, Doderlein non ne aveva ottenuti e nessun altro ci era riuscito: ormai la maggior parte degli ornitologi riteneva che il francolino fosse estinto. Intervenne Doderlein (1871) spiegando che la specie era "comunque prossima ad estinguersi pure ne esisteva tuttora qualche individuo" nella zona di Gela citando le catture del 1865 e del 1869. Salvadori (s.d.: 187), pur non tornando sugli ipotetici esemplari ricevuti dal Museo di Palermo, indicò che "forse" l'ultimo era stato ucciso nell'autunno del 1869, e che "essendo troppo recenti le date delle ultime catture e mi pare che troppo prematuramente si sono affrettati a proclamarne l'estinzione in Sicilia, Lord Lilford ed il Saunders". Doderlein (1874) chiuse definitivamente la questione: nonostante i premi promessi non aveva ottenuto alcun francolino, era evidente che era estinto e l'ultimo era quello ucciso nell'autunno 1869.

Lilford incontrò a Genova il marchese Doria che pure dubitava dell'estinzione del francolino in Sicilia e gliene mostrò un esemplare, acquisito impagliato intorno al 1870, di cui però non si conosceva la data di cattura; incontrò poi Doderlein al Museo di Palermo dove erano conservate due coppie uccise prima del 1870 (Lilford, 1875: 7-24): una "probabilmente [...] avuta tra il 1865 ed il 1869 da Agrigento, e catturata" qualche anno addietro nei dintorni di Gela, l'altra "costituita di un maschio di Agrigento ed una femmina di Palermo, quest'ultima è forse [...] avuta dal conte Ferrero nel 1865" (Arrigoni Degli Oddi, 1929: 807).

Forse però la storia del francolino in Sicilia non era conclusa: ulteriori segnalazioni e catture, ritenute dubbie, si registrarono intorno al 1870 nel trapanese tra Castelvetrano e Selinunte e nel 1886 presso Corleone (PA) (Baccetti, 1992). Quest'ultima desta molte perplessità, sia per la collocazione geografica sia per il lungo lasso temporale intercorso dalle ultime segnalazioni certe. Al contrario, quella relativa al trapanese potrebbe avere fondamento: cronologicamente è prossima agli ultimi dati accertati e la zona era stata una delle due ultime roccaforti del francolino in Sicilia.

La rapida scomparsa della specie è imputabile soprattutto alla "completa mancanza di protezione di esso da parte della legge e delle autorità, specialmente nel periodo posteriore al 1860. Ciò non fa onore ai governi che succedettero all'unificazione, i quali non ebbero pel francolino nessuna delle cure dei governi precedenti" (Amari 1937). Alle carenze della legislazione si sommò l'azione delle bonifiche che privò il francolino degli ambienti umidi di macchia indispensabili alla sua sopravvivenza (Andreotti et al,. 2001: 136).

Nei primi anni Settanta del Novecento, Giovanni Sorci, direttore dell'Osservatorio Ornitologico Siciliano, valutò la possibilità di reintrodurre la specie in Sicilia. Il programma prevedeva l'introduzione sulle isole minori per evitare la dispersione degli individui rilasciati e renderne più semplice lo studio ed il controllo. Vennero identificate idonee: Linosa, Marettimo, Stromboli, Ustica ed alcune isole dell'arcipelago Toscano, le Ponziane e le Tremiti. Il Laboratorio di Ricerche Tecnologiche di Roma avrebbe messo a disposizione dei Comitati Provinciali della Caccia, gratuitamente, i capi da liberare a condizione che le isole prescelte fossero dichiarate oasi (Mazzanti, 1971: 40). Un tentativo venne effettuato alle Egadi (Antoniani, 1972), ma le reintroduzioni in Sicilia non ebbero successo (Brichetti, 1985: 24).

\section{L'ITALIA MERIDIONALE}

Nel Napoletano il francolino "era stato importato dalla Sicilia, e strettamente protetto nelle riserve reali", ma intorno alla metà dell'Ottocento se ne era persa la memoria (Lilford, 1862: 356). Dalla Sicilia era stato introdotto anche in Calabria (Temminck, 1815: 347; Arrigoni Degli Oddi, 1929: 806; Amari, 1937: 1083) dove, lungo la costa ionica della provincia di Reggio, era presente fino alla metà dell'Ottocento. Intorno al 1857 ne furono uccisi due 
a Gerace dove venivano chiamati "pernice gallo", termine che successivamente venne utilizzato per le coturnici da richiamo (Sofia Moretti, 1882; De Fiore, 1889; Arrigoni Degli Oddi, 1902: 338). In quegli anni le leggi del Regno delle Due Sicilie proteggevano i nidi dei francolini nei "domini al di qua del faro", ovvero nella parte continentale del regno (Dias, 1856: 300).

\section{L'ITALIA CENTRALE}

Tutte le popolazioni di francolini introdotte e naturalizzate in Italia continentale a partire dal tardo-medioevo si presume siano originate dalla popolazione siciliana (Andreotti et al., 2001: 136). A Roma nella prima metà del XVII secolo i francolini erano allevati, a scopo alimentare e medicinale, “nell'Ucceliera dell'Illustrissimo Cardinal Borghese nella Vigna di Porta Pinciana" (Olina 1622: 33).

In Toscana, l'introduzione risalirebbe secondo alcuni al 1365 (Arrigoni Degli Oddi, 1929: 806), secondo altri alla fine del XVII sec. (Lavauden, 1936: 306). Dalla documentazione esaminata risulta che furono i Medici a far "trasportare dalla Sicilia nei loro Stati un gran numero di francolini" (Encyclopédie, 1784: 50) ed è probabile che sia stato Lorenzo de' Medici, nella seconda metà del XV sec., ad introdurre la specie, come sembra indicare una lettera di Michele Verino, scritta prima del maggio 1487, nella quale si menzionano uccelli importati nella tenuta di Poggio a Caiano (FI) dalla Sicilia (Masseti, 2008: 212).

Nei secoli XVI e XVII i principi toscani protessero i francolini nelle loro bandite (Savi, 1829: 189) e, sulla base delle normative, si sono individuate quelle che li ospitavano lungo la valle dell'Arno, tra Firenze e Pontedera, e sulle colline fiesolane. L'ottima gestione consentì la diffusione del francolino nelle aree collinari adiacenti e nel Mugello (Baldacci, 1964; Masseti, 2008: 213), ma già all'inizio dell'Ottocento la specie era considerata rara (Sceembri, 1843: 19) se non rarissima o forse quasi del tutto estinta (Savi, 1829: 189). Non è da escludere che, tra Sette e Ottocento, le bonifiche, specialmente nella valle dell'Ombrone a Poggio a Caiano, abbiano giocato un ruolo determinante nella sua scomparsa. Gli ultimi francolini sopravvissero, non molto oltre la metà del secolo, nella zona di Artimino, presso Signa (FI) (Savi, 1829: 189), da dove proviene un esemplare, ucciso in quegli anni, conservato a Firenze (Arrigoni degli Oddi, 1929: 807). Tuttavia ancora nell'ottobre 1859, subito dopo l'annessione della Toscana al nascente Regno d'Italia, la nuova tariffa dei dazi di consumo per le città di Firenze, Pisa, Siena, Lucca e Pistoia citava espressamente i francolini (Bollati, 1866: 310), forse ne vivevano ancora e l'ultimo abbattimento, sembra sia avvenuto nel 1889 (Perco, 1981). La notizia, diffusa nel 1891 ed accolta con molto scetticismo, parlava di una femmina adulta, preparata in pelle, che un tassidermista romano aveva avuto "due anni prima in inverno" da un contadino di Rignano Flaminio, in provincia di Roma (Lepri, 1891: 86). È curioso notare che poco prima di questa segnalazione era stata pubblicizzata un'offerta commerciale di francolini "Chi desidera averne di belli esemplari montati costano L.30, in pelle L.18" (Brogi, 1891: 24).
Nel Novecento in Toscana si effettuarono alcune reintroduzioni. Nel 1932 a Coiano, presso Castelfiorentino (FI), ci fu un primo tentativo, che fallì, perché nella primavera successiva i francolini non nidificarono (Barchielli, 1933). Tra il maggio 1960 e l'aprile 1961 a Miemo, presso Volterra (PI), venne effettuato il lancio di 72 coppie di francolini del Nepal occidentale (ssp. asiae Bonaparte, 1856). Il tentativo ebbe successo e la specie si diffuse nelle zone limitrofe (Baldacci, 1964; Videsott, 1964; R.T., 1967; Perco, 1981, Brichetti, 1985: 24) e, seppure su un esiguo areale, risulta tuttora, dopo un cinquantennio, ben radicata nella zona (Ruggieri, 2006; Puglisi et al., 2009: 426). Nel 1961 venne sperimentata la riproduzione del francolino anche a Capalbio ed al Circeo (Mazzanti, 1971: 38) e se ne tentò l'introduzione nell'Oasi di Palo Laziale (Pratesi, 1983; Brichetti, 1985: 24).

\section{L'ITALIA SETTENTRIONALE}

In Lombardia non erano emerse notizie di presenza della specie se non la citazione settecentesca del Giovio (1795: 88) che, parlando di Como, elogia come la più squisita delle cacciagioni "il francolino Attagen", ma aggiunge "molti ne mandano a noi i monti della Valtellina" evidenziando così la confusione nella determinazione.

Il Ducato di Milano fu sottoposto alla corona spagnola per quasi due secoli (1535-1706), non è quindi del tutto improbabile che i dominatori possano avervi introdotto il francolino che tanto apprezzavano in patria. In effetti alcune notizie farebbero supporre che $i$ francolini possano essere stati importati anche nella Lombardia spagnola. In Brianza, tra Milano e Lecco, due toponimi avvalorano questa ipotesi: Cascina Francolino, storicamente Bicocca del Francolino, in comune di Bernareggio (MB), di epoca riconducibile alla fine del XVI secolo (SIRBeC scheda ARL, 2002-2014) e Cascina Francolino, in comune di Rovagnate (LC), già così denominata nel 1597 (Perego, 1994: 58). Le due località, a quote comprese tra i 200 ed i $350 \mathrm{~m}$ slm e non lontane dall'Adda, potrebbero essere state un habitat idoneo per la specie: la prima è in una zona di campi, la seconda in una valletta paludosa (Boselli, 1993: 138). Un ulteriore labile indizio emerge da una fotografia di un dipinto votivo su tela, ora scomparso, che si trovava a Bernareggio ed era denominato "Madonna del Francolino", nel quale la Vergine Immacolata reggeva in mano una non meglio identificabile macchia nera (Carozzi, 1995). Anche nella Bassa milanese, a circa $90 \mathrm{~m}$ slm tra il Lambro meridionale e quello settentrionale, in comune di Carpiano (MI), un'altra Cascina Francolino, sembra poter alimentare l'ipotesi.

Oltre a questi indizi di possibile presenza durante la dominazione spagnola, un dipinto a olio su tela, che una perizia $^{3}$ attribuisce alla scuola lombarda di fine Seicento, ritrae, in una natura morta, un francolino maschio tra un fagiano, una beccaccia e altri uccelli (Fig. 2). Non pare quindi improbabile che anche in Lombardia il francolino venisse ritratto come negli altri contesti dove la specie era presente nello stesso periodo: al Museo del Prado sono conservate due nature morte con francolini, della prima metà del XVII secolo, una di Tomás Yepes e l'altra di Fe- 


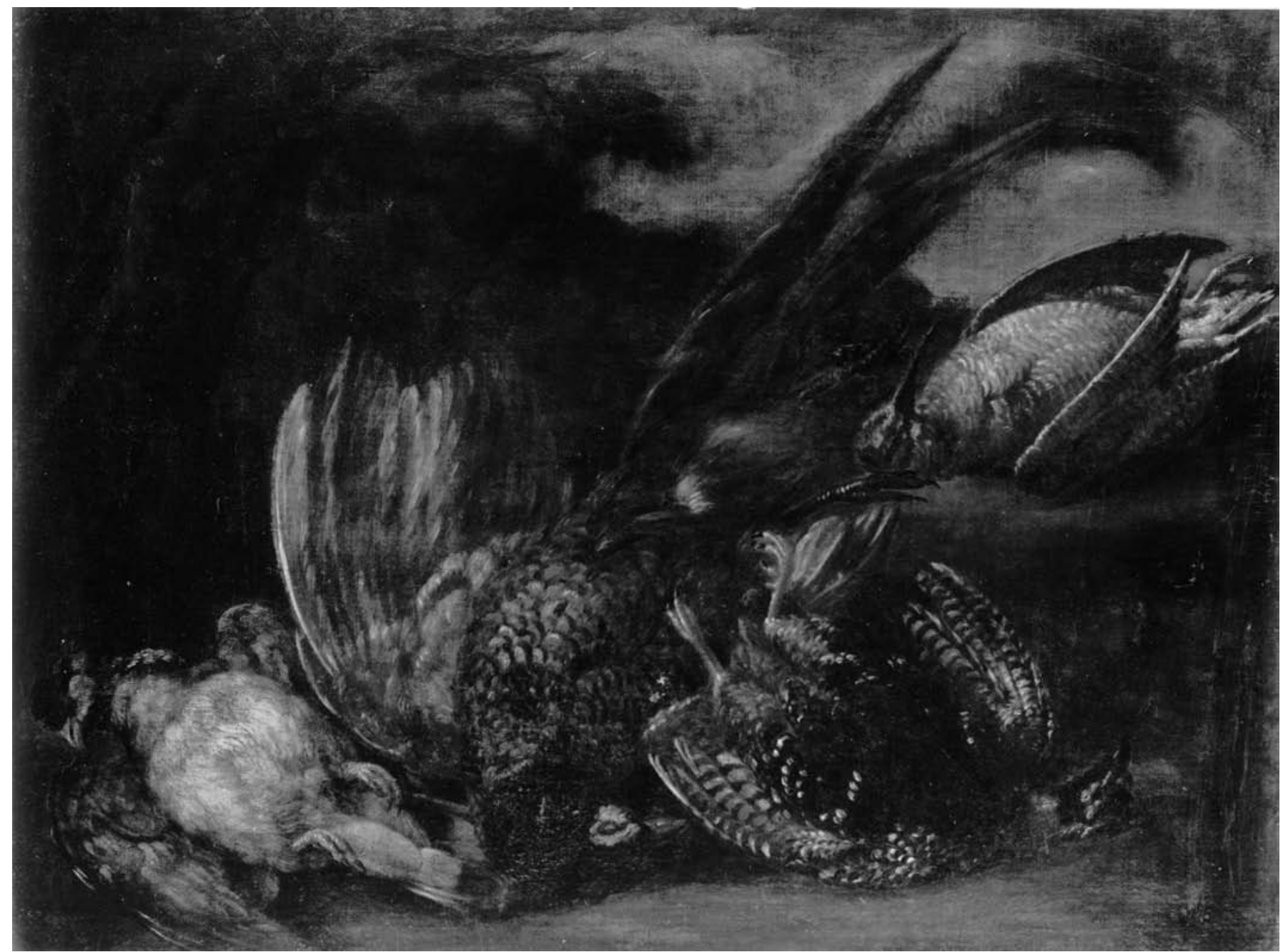

Fig. 2 - "Natura morta con cacciagione", olio su tela Scuola lombarda della fine del XVII secolo. / "Still life with game birds", oil on canvas Lombard school end of $17^{\text {th }}$ century.

lipe Ramirez, ed alla Galleria Palatina di Firenze un dipinto di Justus Sustermans, dello stesso periodo, ritrae dei cacciatori con un francolino.

Un francolino maschio è raffigurato anche in una delle tele del ciclo di Orfeo, attribuite a un non meglio precisato Maestro di Palazzo Lonati Verri che operò a Milano nella seconda metà del XVII sec., ora collocate nella sala detta del Grechetto a Palazzo Sormani a Milano.

In Veneto è nota solo la citazione del XIV secolo di Fazio degli Uberti (Monti, 1826: 209) per la zona di Verona e Vicenza, ma il fatto che sia collegata a quella del gallo cedrone fa supporre che si tratti del francolino di monte ${ }^{1}$. Nei primi anni Ottanta del secolo scorso, il francolino nero venne introdotto in una zona collinare presso Verona, ma fu ben presto eliminato (Baccetti, 1992).

\section{LA SPAGNA}

Il primo documento che ne attesta con certezza la presenza è la "Compilacion de Canellas o de Huesca", raccolta di leggi del Regno d'Aragona compilata nel 1247 per ordine di Giacomo I (1208-1276). Nel capitolo "de venatoribus" è citato il francolino, ma non si può escludere che fosse menzionato anche nelle norme precedenti sulle quali si basava quel compendio (Jiménez, 2013: 17). Si evince che l'introduzione in Spagna non è connessa alla conquista della Sicilia da parte dei Catalano-Aragonesi, avvenuta nel 1282, e tanto meno a quella dei ducati greci di Atene e Neopatria, conquistati tra il 1311 ed il 1319: è quindi plausibile ritenere che i francolini fossero stati importati direttamente dal Mediterraneo orientale. Giacomo I intratteneva rapporti con la Terra Santa e nel 1269 aveva inviato una spedizione in soccorso della città di Acri che, anche grazie al suo aiuto, rimase l'ultimo baluardo cristiano fino al 1291.

Nel corso dei secoli seguenti, il francolino venne diffuso su ampi territori e la sua tutela divenne oggetto di particolari normative. Nel 1368 Pietro IV d'Aragona (1319-1387) inviò francolini e fagiani a Maiorca, che era stata conquistata nel 1229 , perché fossero immessi nella tenuta di Esporlas, vicino a Palma (Maluquer \& Travé, 1961: 145-146), non sembra tuttavia che l'esperimento riuscisse (Jiménez, 2013: 19). Le "Constitucions de Ca- 
thalunya" del 1456 stabilivano che tra il carnevale e la fine di settembre fosse proibita la caccia alle pernici, ai fagiani e ai francolini e fino al 1528 vari provvedimenti di tutela si susseguirono a Teruel, Zaragoza e Calatayud (Jiménez, 2013: 18).

Al di fuori del Regno d'Aragona la documentazione è meno copiosa: a Murcia, riconquistata dai catalani, il francolino era comune e nel 1447 venne proibito agli ebrei e agli arabi di acquistarne nei mercati cittadini (Jiménez, 2013: 18). Giovanni II d'Aragona (1397-1479), ne decretò la completa protezione nel 1454 vietando qualsiasi tipo di caccia, ad esclusione di quella col falcone, nel raggio di una lega da Murcia per consentirne la diffusione (Jiménez, 2013: 19). Qualche anno dopo, nel 1458, il Consiglio di Murcia donò al nuovo re Enrico IV di Castiglia e Leon (1425-1474) quindici coppie di francolini per ripopolare i dintorni del palazzo reale e potervi esercitare la caccia col falcone (Del Carmen Zamora, 1997). La specie era presente, e protetta, nel 1562 anche nella zona di Albacete (Jiménez, 2013: 19) e tra il 1572 ed il 1585 è documentata nelle riserve reali madrilene di El Pardo, Aranjuez e El Escorial dove il francolino era apprezzato da Filippo II (1527-1598). Nel Seicento la specie era comune negli ambienti umidi litorali della costa catalana e valenciana ed il francolino veniva menzionato in un editto del 1671 (Chapman \& Buck, 1910: 321).

Nella prima metà del Settecento, pur cominciando a diventare meno comune in molte zone, risultava ancora abbondante negli ambienti più favorevoli. In Catalogna nel 1706 furono definiti i periodi annuali di chiusura della caccia al francolino. Nel 1722 i francolini erano presenti, ma più rari delle pernici, nella Dehesa, la lingua di terra boscosa tra la laguna dell'Albufera e il mare (Lilford, 1862: 354), ed a metà del secolo viveva ancora nel territorio di Tortosa, in particolare nel delta dell'Ebro, ideale per la specie, e nelle piane di Lérida. Dalla metà del Settecento le popolazioni cominciarono a diminuire e nel 1766 ci si lamentava che "ce ne erano molti al principio del secolo scorso nel Principato [...] Attualmente non ce ne sono più così tanti e nelle altre Province spagnole si trovano appena" (Aymerich in Maluquer \& Travé, 1961: 141). Sul finire del secolo scomparve da quasi tutti i territori abitati in precedenza e la sua storia, costantemente documentata dal XIII secolo sulla base dei provvedimenti di protezione, e la sua caccia, esclusivo diritto dei potenti (Del Carmen Zamora, 1997), stavano finendo per l'inosservanza delle norme di tutela e per le alterazioni ambientali. Scomparve dall'Aragona durante il XVIII secolo e nella prima metà dell'Ottocento anche dai pochi distretti di rifugio in cui era sopravvissuto: il delta dell'Ebro, l'Albufera di Valencia e le piane della provincia di Lérida. A Valencia si estinse tra il 1825 ed il 1830 ed il museo universitario di quella città conservava alcuni esemplari della Dehesa (Lilford, 1862: 354) che andarono persi in un incendio (Maluquer \& Travé, 1961: 148). Nelle piane di Lérida, intorno al 1840 , furono uccisi gli ultimi francolini che vi avevano trovato rifugio nelle zone poco popolate e meno coltivate rispetto alla costa. Nel 1845 il francolino era ancora menzionato tra le specie cacciabili (Hidalgo \& Gutierrez Gonzalez, 1845: 106-107) e forse sopravvisse ancora nelle piane umide di Ampurdán, nella zona di Figueres in provincia di Gerona, fin oltre la metà dell'Ottocento (Maluquer \& Travé, 1961: 143).

L'ipotesi di presenza a Minorca è molto debole basandosi unicamente su di un esemplare che era conservato al museo di Mahón. Ancora più ipotetica la presenza ad Ibiza dove l'unica prova consiste in un francolino raffigurato nel retablo di una chiesa (Maluquer \& Travé, 1961: 146). L'Andalusia, rimasta sempre fuori dall'influenza aragonese, non ha mai ospitato francolini, nonostante gli habitat ottimali (Maluquer \& Travé, 1961: 149) e gli autori arabi non sembrano avene mai menzionato la presenza (Jiménez, 2013: 20). Così pure era del tutto assente dagli altri vasti territori dominati dagli arabi: Maiorca, Murcia e Valencia dove la specie comparve solo dopo la "reconquista".

Negli anni Novanta del secolo scorso si tentò la reintroduzione a Maiorca (Jiménez, 2013: 21) ed altri rilasci furono effettuati anche in Catalogna, sul basso corso del fiume Cinca, ed in Portogallo (Matias, 2002).

\section{LA FRANCIA}

In Francia la sola regione dove è accertata la presenza storica della specie è quella sud-occidentale, che fu parte del Regno d'Aragona, e poi di quello di Spagna, fino al 1660 .

Il primo documento che cita il francolino nel Rossiglione è una lettera di Eleonora di Sicilia (1325-1375), terza moglie di Pietro IV d'Aragona (1319-1387), inviata da Perpignan nel 1351, con la quale chiedeva che le fosse inviata dalla Sicilia una coppia di francolini (Jiménez, 2013: 18). Da allora vari documenti testimoniano la costante presenza della specie: all'inizio del XVII secolo viveva numerosa nella pianura costiera ricca di corsi d'acqua e stagni litorali (Maluquer \& Travé, 1961: 141) e nel 1628 era menzionata ancora tra quelle cacciabili nel Rossiglione (Maluquer \& Travé, 1961: 145). Relativamente alla Provenza le notizie si basano solo su citazioni molto antiche e confuse, sembrerebbe fosse presente verso la metà del XVI sec. forse nella Camargue che tuttora rappresenta un ambiente ideale per il francolino (Lavauden, 1936: 308; Maluquer \& Travé, 1961: 136).

\section{CONCLUSIONI}

Il francolino, dalle coste orientali del Mediterraneo, venne importato in Sicilia ed in Spagna intorno alla metà del XIII secolo e successivamente, da queste zone, fu introdotto in Toscana, e forse anche in Lombardia, ma in quest'ultima regione finora non sono state condotte indagini approfondite. Le motivazioni di queste operazioni furono essenzialmente di carattere venatorio e fino a che perdurarono le grandi riserve, con le relative norme di tutela della selvaggina, la sopravvivenza e la diffusione del francolino furono garantite. Tra la fine del Settecento e la metà dell'Ottocento, il frazionamento dei latifondi e la progressiva bonifica e messa a coltura dei terreni portarono alla rapida scomparsa della specie che, per le sue caratteristiche, non poteva sopravvivere 
a radicali modifiche ambientali ed alla caccia non regolamentata. Quelle stesse ragioni, tra metà Ottocento e i primi decenni del Novecento, portarono il francolino all'estinzione anche in quasi tutte le pianure costiere del Mediterraneo orientale.

\section{Ringraziamenti}

Si ringraziano Giulia Poma e Federico Oriani della Società Italiana di Scienze Naturali per la collaborazione nella stesura del testo; Ambrogio Rossi per aver gentilmente fornito i dati e l'immagine del dipinto di scuola lombarda del XVII secolo; Lorenzo Calì del Museo Regionale di storia naturale di Terrasini (PA) e Roberto Pazzi del Museo di scienze naturali "T. Salvadori” di Fermo per le informazioni sugli esemplari conservati nelle rispettive collezioni.

\section{NOTE}

${ }^{1}$ Catalano, ceco, danese, greco moderno, inglese, spagnolo, estone, finlandese, francese, italiano, lituano, olandese, norvegese, polacco, portoghese, slovacco, sloveno, svedese, tedesco, ungherese.

${ }^{2}$ Museo Ornitologico Ferrante Foschi di Forlì esemplare $\mathrm{n}^{\circ} 2175$ ex Collezione Benoit (<http://www.arif.it/Museo/ catalogo/sp103.htm>)

${ }^{3}$ Verbale di perizia ${ }^{\circ} 3839$ Istituto Finanziario per l'Arte Finarte spa "olio su tela $\mathrm{cm} 68 \times 87$ Natura morta con cacciagione Scuola lombarda della fine del XVII secolo elencato al n' 203 asta 474 Milano 28 maggio 1984”.

4 “... In quella parte lo Cedron s'ingenera, / La di cui carne è di cotal natura, / Che qual par bue, e qual fagian, si è tenera. / Le penne sue han di pavon figura, / Combatte per amor, e come il cieco / Prender si lascia, tanta è la sua arsura. / Similmente alla mente mi reco / Che la trovasi l'uccello francolino, / ..."

\section{BIBLIOGRAFIA}

AA.VV., 1784 - Encyclopédie Metodique. Histoire naturelle. Oiseaux. Tome II. Paris: Libraire Panckouke.

Amari G., 1937 - Avifauna siciliana scomparsa: il francolino. Diana, 32 (24): 1082-1084.

Andreotti A., Baccetti N., Perfetti A., Besa M., Genovesi P. \& Guberti V., 2001 - Mammiferi e uccelli esotici in Italia: analisi del fenomeno, impatto sulla biodiversità e linee guida gestionali. Quad. cons. Natura. Min. Ambiente, 2.

Andrews I.J., 1995 - The birds of the Hashemite Kingdom of Jordan. Dundee: Burns \& Harris Ltd..

Antoniani A., 1972 - La reintroduzione del francolino alle Egadi. La riserva di caccia, 1: 30.

Arnott W.G., 2007 - Birds in the ancient world from A to Z. New York: Routledge.

Arrigoni degli Oddi E., 1902 - Atlante ornitologico, Uccelli europei con notizie d'indole generale e particolare. Milano: Hoepli.

Arrigoni degli Oddi E., 1929 - Ornitologia italiana. Milano: Hoepli.
Baccetti N., 1992 - 133. Francolino Francolinus francolinus (Linnaeus, 1766) in Fauna d'Italia. Vol. XXIX Aves I: Gaviidae - Phasianidae. Brichetti P., De Franceschi P. \& Baccetti N. (eds.). Bologna: Calderini (pp. 792-799).

Baldacci U., 1964 - Il francolino, sua reintroduzione in Europa. Pisa: Nistri-Lischi.

Bannerman D.A. \& Bannerman M.W., 1958 - Birds of Cyprus. Edimburg: Oliver and Boyd.

Barchielli G., 1933 - Per la caccia e per i cacciatori. In Del francolino volgare. Zenere A., Diana, 34 (23).

Benoit L., 1840 - Ornitologia siciliana. Messina: Fiumara.

Berk (van den) V., 1991 - Impressions of autumn migration in mid-october along the Çukurova coast, near the Belen Pass, southern Turkey. Ornithological Society of the Middle East Bulletin, 26: 16-19.

Bollati E. (a cura di), 1866 - Fasti legislativi e parlamentari delle rivoluzioni italiane nel secolo XIX. Volume II, 1859-1861, Parte II, Toscana. Milano: Giuseppe Crivelli.

Bonaparte C., 1856 - Notes sur les tableaux des Gallinacés. Comptes rendus des séances de l'Académie des Sciences. Tome 43. Séance de 12 mai 1856.

Boselli P., 1993 - Dizionario di toponomastica briantea, comasca e lecchese. Lecco: Stefanoni.

Boye P., 1990 - On the distribution and status of the Black Francolin, Francolinus francolinus, in Cyprus. Zoology in the Middle East, 4: 17-22.

Brichetti P., 1985 - Distribuzione attuale dei Galliformi (Galliformes) in Italia. Atti sem. biologia galliformi, 15-27.

Brisson A.D., 1760 - Ornithologie. Tome I. Paris: J.B. Bauche.

Brogi S. (a cura di), 1891 - Offerte. Bollettino del naturalista, collettore, allevatore, coltivatore 11 (2): 24.

Buffon (conte di) Leclerc G.L., 1775 - Storia naturale degli uccelli. Tomo IV. Milano: Galeazzi.

Carozzi D., 1995 - Bernareggio usi e costumi della sua gente. Bernareggio: Sip.

Carvana G., 1889 - Francolino. In Primo resoconto dei risultati della inchiesta ornitologica in Italia. Parte I: Avifauna italica, elenco sistematico delle specie di uccelli stazionarie o di passaggio in Italia. Giglioli H.E. (a cura di). Firenze: Le Monnier (pp. 516-520).

Chapman A. \& Buck W.J., 1910 - Unexplored Spain. edizione 1978. Madrid: Incalfo.

Costa O.G., 1857 - Fauna del Regno di Napoli. Uccelli Parte I. Napoli: Gaetano Sautto.

Crivella A., 1593 - Trattato di Sicilia in Federico II di Svevia. De arte venandi cum avibus. Trombetti $\mathrm{Bu}-$ driesi A.L. (a cura di). Bari: Laterza (nota 112 alla Introduzione).

Cuvier G. (a cura di), 1828 - Caii Plinii Secundi, Historiae Naturalis Libri XXXVII, Pars Tertia continens zoologiam, Volumen quartum. Paris: Nicolaus Eligius Lemaire.

De Fiore C., 1889 - Francolino. In Primo resoconto dei risultati della inchiesta ornitologica in Italia. Parte I: Avifauna italica, elenco sistematico delle specie di uccelli stazionarie o di passaggio in Italia. Giglioli H.E. (a cura di). Firenze: Le Monnier (p. 515). 
Del Carmen Zamora M., 1997 - Aprovechamientos forestales en la Comarca del Campo de Cartagena durante la Edad Media. Scripta Nova. Revista Electrónica de Geografía y Ciencias Sociales, 13.

Dias F., 1856 - Amministrazione finanziera del Regno delle Due Sicilie. Napoli: Giovanni Pellissone.

Doderlein P., 1869 - Avifauna del modenese e della Sicilia. Giornale di Scienze naturali ed economiche: 5.

Doderlein P., 1871 - Avifauna del modenese e della Sicilia. Fascicolo terzo. Giornale di Scienze naturali ed economiche: 7: 9-72.

Doderlein P., 1874 - Avifauna del modenese e della Sicilia. Fascicolo sesto. Giornale di Scienze naturali ed economiche: 10: 329-381.

Dresser H.E., 1877 - A History of the Birds of Europe including all the species inhabiting the Western Palæarctic Region. Vol. VII. London: Published by the author.

Drummond H.M., 1846 - List of the birds observed to winter in Macedonia. The annals and magazine of natural history, 18 (116): 10-15.

Falcone H., 1675 - Narciso al fonte. Venezia: Hertz.

Festa E., 1894 - Viaggio del dr. E.Festa in Palestina, nel Libano e regioni vicine. Boll. Musei di zoologia ed anatomia comparata della R. Università di Torino, 9 (172)

Forcina G., Panayides P., Guerrini M., Nardi F., Gupta B.K., Mori E., Al-Sheikhly O.F., Mansoori J., Khaliq I., Rank D.N., Parasharya B.M., Khan A.A., Hadjigerou P. \& Barbanera F., 2012 - Molecular evolution of the Asian francolins (Francolinus, Galliformes): a modern reappraisal of a classic study in speciation. Molecular Phylogenetics and Evolution, 65: 523534.

Galloni P., 1993 - Il cervo e il lupo. Caccia e cultura nobiliare nel Medioevo. Bari: Laterza.

Garcia Soler M.J., 2003 - La alimentació y la salud en la Grecia antigua. <http://www.academia.edu/2442358/ La_alimentacion_y_la_salud en_la_Grecia_antigua>

Gioviō G.B., 1795 - Como e il Lario. $\overline{\text { E}}$ dizione 1999. Milano: Valentina Edizioni.

Hartert E., 1917 - Notes on game-birds. Novitates Zoologicae, 24: 275-292.

Hildago C. \& Gutierrez Gonzalez A., 1845 - Tratado de caza. Madrid: Manuel Alvarez.

Hollom P.A.D., Porter R.F., Christensen S. \& Willis I., 1988 - Birds of the Middle East and North Africa. Calton: T. \& A. D. Poyser.

Houlihan P.F., 2002 - Animals in Egyptian Art and Hieroglyphs. In A history of animal World in the ancient Near East. Collins B.J. (ed.). Leiden: Brill (pp. 97144).

Hüe F. \& Etchécopar R.D., 1970 - Les oiseaux du proche et du moyen orient. Paris: N. Boubée \& Cie.

James, 1753 - Dizionario universale di medicina. Tomo terzo. Venezia: Giambatista Pasquali.

Jiménez J., 2013 - El curioso caso del francolin: extinguido tras 600 años de presencia en España. Quercus, 330: 16-23.

Kirwan G.M. \& Martins R.P., 1994 - Turkey bird report 1987-91. Sandgrouse, 16 (2): 77-117.
Kirwan G., Demirci B., Welch H., Boyla K., Özen M., Castell P. \& Marlow T., 2008 - The Birds of Turkey. London: Christopher Helm.

Labat J.B., 1735 - Memoires du Chevalier D'Arvieux. Tome I. Paris: C.J.B. Delespine.

Latham J., 1783 - A general synopsis of birds. Vol. II part 2. London: Leigh \& Sotheby.

Lavauden L., 1936 - Les Francolins. Alauda, 8 (3-4): 301-315.

Lepri G., 1891 - Sopra un Francolinus vulgaris ucciso nella provincia di Roma. Bollettino del naturalista, collettore, allevatore, coltivatore, 11 (7): 86-87.

Lilford Lord (Thomas Littleton Powys), 1862 - On the extinction in Europe of the common francolin. Ibis, 4: 352-354.

Lilford Lord (Thomas Littleton Powys), 1875 - Cruise of the "Zara" in the Mediterranean. Ibis, III series, 5 (17): $1-35$.

Linnaeus C., 1766 - Systema naturae. Per regna tria naturae, Tomus I. Laurentii Salvii: Holmiae.

Malherbe A., 1843 - Faune ornithologique de la Sicile. Metz: Typographie de S. Lamort.

Maluquer J. \& Travé F., 1961 - Presencia y extinción del francolín en la peninsula ibérica e islas Baleares. Ardeola, 7: 135-156.

Mariti G., 1769 - Viaggi per l'isola di Cipro e per la Soria e Palestina fatti da Giovanni Mariti fiorentino dall'anno MDCCLX al MDCCLXVIII. Tomo I. Lucca: Jacopo Giusti.

Martins R.P., 1989 - Turkey bird report 1982. Sandgrouse, 11: $1-41$.

Martorelli G., 1906 - Gli uccelli d'Italia. Terza edizione (1960) riveduta ed aggiornata da Edgardo Moltoni e Carlo Vandoni. Milano: Rizzoli.

Masseti M., 2008 - Uomini e (non solo) topi. Firenze: Firenze University Press.

Masseti M., 2009 - In the gardens of Norman Palermo, Sicily (twelfth century A.D.). Anthropozoologica, 44 (2): 7-34. < http://www.bioone.org/doi/full/10.5252/ az2009n2a1>.

Matias R., 2002 - Lista das aves exóticas observadas em liberdade em Portugal continental. Lisboa: Instituto da Conservação da Natureza Sociedade Portuguesa para o Estudo das Aves.

Mazzanti C., 1971 - Il francolino e il ripopolamento del mezzogiorno. Diana, (9): 37-40.

Mayaud N., 1939 - Le francolin a-t-il existé en Corse? Alauda, 11: 65-67.

McGowan P.J.K., 1994 - Family Phasianidae (Pheasants and Partridges). In Handbook of the Birds of the World. Vol. 2 New World Vultures to Guineafowl. Del Hoyo J., Elliot A. \& Sargatal J. (eds.). Barcelona: Lynx Edicions (pp. 434-552).

Meinertzhagen R., 1920 - Notes on the birds of Southern Palestine. Ibis, $11^{\text {th }}$ series, 2: 195-259.

Meinertzhagen R., 1933 - Descriptions of new subspecies of Wren and Francolin. Bulletin of the British Ornithologists' Club, 54: 20-21.

Minà-Palumbo F., 1889 - Francolino. In: Primo resoconto dei risultati della inchiesta ornitologica in Italia. Parte I: Avifauna italica, elenco sistematico delle 
specie di uccelli stazionarie o di passaggio in Italia. Giglioli H.E. (a cura di). Firenze: Le Monnier (pp. 520-521).

Moltoni E., 1946 - L'etimologia ed il significato dei nomi degli uccelli italiani. Rivista italiana di ornitologia, 16: 69-92.

Moltoni E. \& Frugis S., 1967 - Gli uccelli delle Isole Eolie. Rivista italiana di ornitologia, 37: 91-234.

Monti V., 1826 - Il Dittamondo di Fazio degli Uberti. Milano: Giovanni Silvestri.

Mouton-Fontenille M.J.P., 1811 - Traité élémentaire d'ornithologie. Seconde partie. Lion: Yvernault \& Cabin.

Ogilvie-Grant W.R., 1896 - A hand-book to the game birds. Vol. I: Sand-grouse, partridges, pheasant. London: Edward Lloyd.

Olina G.P., 1622 - Ucceliera, ovvero discorso della natura e proprietà di diversi uccelli e in particolare quelli che cantano, con il modo di prenderli, conoscerli, allevarli e mantenerli. Roma: Andrea Fei.

Orlando C., 1936 - Specie nidificanti nella zona litoranea tra i Golfi di Palermo e di Castellammare. Rivista italiana di ornitologia, 6: 201-205.

Perco F., 1981 - Dopo centocinquanta anni il francolino è ritornato a volare in Toscana: ma per la legge non esiste. Airone, 1 (5): 49.

Perego N. (a cura di), 1994 - I luoghi della memoria, Rovagnate e Perego: nei loro nomi la storia della Brianza. Barzanò: Marna.

Pitton de Tournefort J., 1718 - Relation d'un vojage du Levant. Tomo I. Amsterdam: La Compagnie.

Pococke R., 1745 - A description of the East and some other Countries. Vol. II Part I Observations on Palestine or Holy Land, Syria, Mesopotamia, Cyprus and Candia. London: W. Bowyer.

Pratesi F., 1983 - Palo: il bosco, i prati e il parco. Natura Oggi, 1 (8): 96-97.

Puglisi L., Bosi E., Corsi I., Del Sere M., Pezzo F., Sposimo P. \& Verducci D., 2009 - Usignolo del Giappone, Bengalino \& Co: alieni in Toscana. Alula, 16 (1-2): 426-431.

Ramadan-Jaradi G. \& Ramadan-Jaradi M., 1999 - An updated checklist of the birds of Lebanon. Sandgrouse, 21 (2): 132-170.

Roberts O., 1999 - Akamas, Cyprus: migrants and endemics. Sandgrouse, 21 (2): 128-131.

R.T., 1967 - Il francolino, selvatico del Granduca. Diana, 62 (7): 41.

Ruggieri, 2006 - Francolino. In Cronaca ornitologica toscana, osservazioni relative agli anni 1992-2004. Arcamone E. \& Puglisi L. (eds.). Alula, 13 (1-2): 19.

Salvadori T., 1869 - Letter $26^{\text {th }}$ december. Ibis, new series, 6: 153-154.

Salvadori T., s.d. (1871-72) - Fauna d'Italia, Uccelli. Milano: Vallardi.

Saunders H., 1869 - Notes on the ornithology of Italy and Spain. Ibis, new series, 5: 391-403.

Saunders H., 1870 - Letter $21^{\text {st }}$ march 1870 . Ibis, new series, 6: 298-299.

Savi P., 1829 - Ornitologia toscana. Tomo II. Pisa: Tipografia Nistri.
Sceembri A., 1843 - Quadro geografico-ornitologico ossia Quadro comparativo. Le ornitologie di Malta, Sicilia, Roma, Toscana, Liguria, Nizza e la provincia di Gard. Malta.

Shaw M.D., 1743 - Voyages de Monsr. Shaw, M.D. dans plusierurs provinces de la Barbarie e du Levant. Tome I. La Hate: Jean Neaulme.

SIRBeC scheda ARL, 2002-2014 - MI100-00697. Regione Lombardia e Università degli Studi di Pavia. $<\mathrm{http}: / / \mathrm{www}$. lombardiabeniculturali.it/architetture/ schede-complete/MI100-00697/>

Smith T., 1826 - Il gabinetto del giovane naturalista. Tomo IV. Milano: Omobono Manini.

Sofia Moretti F., 1882 - I tetraonidi. La caccia, 7 (170): 1420-1421.

Sonnini de Manoncourt C.S., 1798 - Voyage dans la Haute et Basse Egypte fait par ordre de l'ancien gouvernement. Tome I. Paris: F.Buisson.

St.John J.A., 1844 - The Hellenes: the history of the manners of the ancient greecs. Vol. II. London: Richard Bentley.

Sultana J., Guaci C. \& Beaman M., 1975 - A guide to the birds of Malta. La Valletta: Malta Ornithological Society.

Tanara V., (s.d. XVII sec.) - La caccia degli uccelli. Manoscritto pubblicato a cura di Alberto Bacchi della Lega, 1886. Bologna: Romagnoli-Dall'Acqua.

Temminck C.J., 1815 - Histoire naturelle générale des Pigeons et des Gallinacées. Vol. III. In Le francolin a-t-il existé en Corse? Mayaud N. Alauda, 11: 66.

Tristram H.B., 1865 - The land of Israel; a journal of travels in Palestine. London: Society for promoting christian knowledge.

Tristram H.B., 1868 - On the ornithology of Palestine. VII. Ibis, 4 (14): 204-215.

Tristram H.B., 1884 - The survey of Western Palestine. The fauna and flora of Palestine. London: Committee of the Palestine exploration fund.

Trombetti Budriesi A.L. (a cura di), 2000 - De arte venandi cum avibus di Federico II di Svevia. Edizione e traduzione italiana del ms. lat. 717 della Biblioteca Universitaria di Bologna collazionato con il ms. Pal. lat. 1071 della Biblioteca Apostolica Vaticana. Bari: Laterza.

Tyrberg T., 1998 - Pleistocene birds of the Palearctic: a catalogue. Cambridge (Mass.): Nuttall Ornithological Club.

Videsott R., 1964 - Il ritorno del francolino in Toscana. Diana, 59 (22): 17-22.

Vivaldi C. (a cura di), 1993 - Marziale Marco Valerio. Gli epigrammi. Roma: Grandi Tascabili Economici Newton.

Volney M.C.F., 1801 - Travels in Syria and Egypt, during the years 1783, $1784 \& 1785$. Vol. I., Perth: Morrison R.

Wilde W.R., 1840 - Narrative of a voyage, Madeira, Tenerife and along the shores of the Mediterranean, including Algiers, Egypt, Palestine, Tyre, Rhodes, Telmessus, Cyprus and Greece. Vol. II. Dublin: William Curry Jun. \& co. 